\title{
“NO ME AGRADA VIAJAR". MOVERSE EN LA CIUDAD COMO DESAFÍO COTIDIANO PARA JÓVENES DE BARRIOS POPULARES DE BUENOS AIRES
}

\author{
SILVIA ALEJANDRA TAPIA ${ }^{1}$
}

\begin{abstract}
RESUMEN
El artículo propone vinculaciones entre la sociología de la individuación de Danilo Martuccelli y los estudios sobre movilidades espaciales para indagar las desigualdades en los desplazamientos cotidianos de jóvenes de barrios populares de Buenos Aires. Del análisis de los resultados de una investigación actualmente en curso, se identifica que las movilidades se vuelven pruebas cotidianas que estos jóvenes deben atravesar, dado que gran parte de sus actividades cotidianas se desarrolla fuera de estos barrios. Tales retos suponen un trabajo significativo para ellos, para lo cual movilizan soportes materiales y simbólicos, que expresan desigualdades, pero también estrategias para afrontarlas.
\end{abstract}

PALABRAS ClaVES: JUVENTUDES - MOVILIDADES COTIDIANAS- INDIVIDUACIÓN

\footnotetext{
${ }^{1}$ Dra. en Ciencias Sociales, Magíster en Políticas Sociales y Lic. en Sociología por la Universidad de Buenos Aires. Becaria posdoctoral por el Consejo de Investigaciones Científicas y Técnicas, con lugar de trabajo en el Área de Salud y Población del Instituto de Investigaciones Gino Germani de la Facultad de Ciencias Sociales, Universidad de Buenos Aires (IIGG-UBA). Correo electrónico: tapiasilvi1@gmail.com
} 
202 "No me agrada viajar". Moverse en la ciudad como desafío cotidiano para jóvenes de barrios populares de Buenos Aires

\title{
“NÃO GOSTO DE VIAJAR”. DESLOCAR-SE PELA CIDADE \\ COMO DESAFIO COTIDIANO PARA JOVENS DE BAIRROS \\ POPUlARES DE BUENOS AIRES
}

\begin{abstract}
RESUMO
O artigo propõe vinculações entre a sociologia da individuação de Danilo Martuccelli e os estudos sobre as mobilidades espaciais para indagar as desigualdades nos deslocamentos cotidianos de jovens de bairros populares de Buenos Aires. A partir da análise dos resultados de uma pesquisa atualmente em andamento, são identificadas as mobilidades que se tornam provas cotidianas que estes jovens devem passar, já que grande parte de suas atividades cotidianas são fora destes bairros. Estes desafios supõem um trabalho significativo para eles, para o qual mobilizam suportes materiais e simbólicos, que expressam desigualdades, mas também estratégias para afrontá-las.
\end{abstract}

PALAVRAS-CHAVE: JUVENTUDES, MOBILIDADES COTIDIANAS, INDIVIDUAÇÃO.

\section{“I DON'T LIKE COMMUTING" MOVING THROUGH THE CITY AS A DAILY CHALLENGE FOR YOUNG DWELLERS FROM WORKING CLASS NEIGHBORHOODS OF BUENOS AIRES}

\begin{abstract}
In order to inquire into the inequities in daily commuting of young individuals from working class neighborhoods of Buenos Aires, the present article sets out links between Danilo Martuccelli's sociology of individuation and studies about spatial mobility. From the analysis of data collected from a currently ongoing study, commuting is identified as a daily challenge for these young individuals, as a big part of their everyday activities is developed out of their neighborhoods. Such challenge, implying a big effort to go through by means of material and symbolic support, reflects several inequalities but also the strategies to deal with them.
\end{abstract}

KEYWORDS: YOUTHS, DAILY COMMUTING, INDIVIDUATION. 


\section{INTRODUCCIÓN}

EN ESTE ARTÍCULO presento avances de los resultados de un proyecto de investigación actualmente en curso, ${ }^{2}$ cuyo objetivo general es analizar las experiencias sociales de jóvenes en instituciones que propician el acceso y el ejercicio de derechos en barrios populares del Área Metropolitana de Buenos Aires (AMBA), ${ }^{3}$ indagando sus vinculaciones con sus procesos de individuación. En particular, en este trabajo me propuse interrogar: ¿cómo se vinculan las movilidades cotidianas urbanas de jóvenes que residen en barrios populares del AMBA con los procesos de desigualdad y exclusión social en sus procesos de individuación? ¿Es posible pensar estas movilidades cotidianas urbanas como pruebas sociales estructurales en el contexto de las sociedades latinoamericanas que los jóvenes deben atravesar? ¿Cómo perciben los jóvenes de sectores populares sus movilidades cotidianas y qué estrategias despliegan para transitar, usar y apropiarse del espacio urbano? ¿Con qué soportes cuentan para atravesar el desafío cotidiano de desplazarse y desarrollar diferentes espacios y actividades educativas, laborales, culturales, de cuidado y de sociabilidad?

Guiado por tales interrogantes, el objetivo de este artículo se centra en articular las herramientas conceptuales de la sociología de la individuación y los estudios sobre movilidades para indagar el tipo de (in)movilidades que se producen en las vidas cotidianas de estos jóvenes a fin de identificar el modo en que se vuelven posibles los desplazamientos requeridos para llevar adelante

\footnotetext{
${ }^{2}$ Proyecto Instituciones, derechos e individuación: un análisis de sus vinculaciones en las experiencias sociales de jóvenes en barrios populares del Área Metropolitana de Buenos Aires (UBACYT 2016-2017). Director y co-directora: Dr. Pablo F. Di Leo y Dra. Ana Josefina Arias (IIGG-UBA).

${ }^{3}$ El AMBA está comprendido por la Ciudad Autónoma de Buenos Aires (CABA) y 24 municipios que la rodean en el denominado Conurbano bonaerense o Gran Buenos Aires (GBA). Constituyen los aglomerados urbanos más importantes de Argentina, con índices altamente diferenciales en su interior (Maceira, 2012).
} 
204 "No me agrada viajar". Moverse en la ciudad como desafío cotidiano para jóvenes de barrios populares de Buenos Aires

prácticas y vínculos en sus cotidianeidades. Se entiende que advertir las maneras en que se perciben y se ponen en práctica diversas formas de movilidad cotidiana por el espacio urbano -frente a los abordajes de los procesos de desigualdad reducidos a espacios delimitados y estáticos (Jirón, Lange y Bertrand, 2010)- permite reconocer y problematizar las prácticas que incluyen y excluyen, regulando o potenciando los desplazamientos, habilitando u obstaculizando la movilización de soportes materiales y simbólicos a los que jóvenes que residen en barrios populares pueden acceder. Y, en consecuencia, considerar el análisis de estas (in)movilidades como dimensiones sustantivas para comprender procesos de desigualdad y exclusión social, pero también como potenciadoras de resignificaciones y resistencias frente a éstas.

La investigación siguió una estrategia metodológica de tipo cualitativo, respondiendo a un diseño abierto y flexible (Vasilachis de Gialdino, 2012). Para la construcción de los datos empíricos se realizaron entrevistas ${ }^{4}$ en profundidad a jóvenes ${ }^{5}$ y referentes de instituciones en barrios de la zona sur del AMBA que, en investigaciones previas, fueron identificadas como significativas en las vidas de los jóvenes (Di Leo y Camarotti, 2013; 2015). En este trabajo, se analizan las entrevistas a los jóvenes según los lineamientos generales de la teoría fundamentada. A partir de la estrategia de la comparación constante, fueron codificadas y analizadas, considerando las dimensiones indagadas y otras temáticas emergentes (Glaser y Strauss, 1967).

La muestra (ver Anexo I) estuvo compuesta por 14 jóvenes, de entre 14 y 24 años, que concurrían a diversas instituciones. Una escuela secundaria pública del GBA y dos bachilleratos populares -uno en CABA y otro en

\footnotetext{
${ }^{4}$ Durante el desarrollo del trabajo de campo se tomaron los resguardos éticos para preservar el anonimato, la identidad y la integridad de los participantes en las entrevistas, de manera informada y voluntaria, asegurando la confidencialidad de sus respuestas.

${ }^{5}$ Teniendo conciencia de la orientación androcéntrica del español, utilizamos el género masculino en los plurales sólo para facilitar la lectura.
} 
GBA-, esto es, “(...) espacios desarrollados por organizaciones sociales, agrupaciones de estudiantes universitarios, cooperativas de docentes, [que] tras su creación buscaron el reconocimiento estatal de esta acción. Se trata de espacios de escolarización secundaria para jóvenes y adultos que tomando como referencia a los CENS (Centro Educativo de Nivel Secundario), organizan una graduación de tres ciclos" (García, 2013, p.1). Una cooperativa de trabajo (en adelante COP) que, con subsidio estatal, ofrece cursos de formación en oficios (electricidad, mecánica, herrería, etc.), espacios de recreación y refrigerios en la CABA. Y por último, un circo, organización social que, desde 1998 en CABA, ofrece talleres de artes circenses, hip-hop, parkour, kung-fu, maquillaje, fundamentalmente a partir de la participación en un programa estatal. Tiene como propósito contribuir a la transformación personal, comunitaria y social a través del arte (Tapia, 2016).

En todas estas instituciones se ofrecen espacios de formación y recreación gratuitos cuyos principales destinatarios son jóvenes. Los barrios en los que éstos residen y donde se ubican dichas instituciones se sitúan en el sur y oeste del AMBA, en áreas de mayor empobrecimiento urbano. En particular, en villas y asentamientos. Si bien presentan diferencias entre sí, en rasgos generales, refieren a urbanizaciones informales resultantes de ocupaciones de terrenos vacantes o privados, deshabitados o baldíos. Registran alta densidad poblacional, viviendas construidas con materiales precarios y escaso acceso a servicios públicos o espacios verdes y/o recreativos. Sus habitantes son trabajadores informales o con baja calificación, que suelen ser estigmatizados socialmente. Fueron creados a partir de la década de los 40 y 50 por los mismos pobladores en la búsqueda de tener un lugar (Cravino, Del Río y Duarte, 2000; Machado, Martiñan y Grinberg 2016).

La exposición del trabajo se organiza de la siguiente manera: primero, se despliegan las herramientas analíticas a partir de las conceptualizaciones de la sociología de la individuación, los estudios sobre juventudes y los estudios 
206 "No me agrada viajar". Moverse en la ciudad como desafío cotidiano para jóvenes de barrios populares de Buenos Aires

sobre movilidades. Luego, en diálogo con el marco conceptual, se analizan los emergentes en los relatos de los jóvenes donde se identificaron tres dimensiones relevantes en sus movilidades cotidianas: la articulación de obligaciones y opciones cotidianas, las posibilidades y limitaciones para moverse 0 permanecer en diferentes áreas urbanas y las significaciones y sentires ${ }^{6}$ acerca de las mediaciones técnicas para realizar tales desplazamientos. En la sección final, se recuperan la articulación entre las dimensiones y categorías propuestas y se reflexiona sobre los aportes del abordaje de las movilidades al análisis de las experiencias juveniles.

\section{JUVENTUDES, INDIVIDUACIÓN E (IN)MOVILIDADES}

En los últimos años se ha remarcado el carácter situado y relacional de las juventudes. Así, frente a los discursos que entendieron la juventud como un período vital homogéneo e invariable, se destacó la complejidad y diversidad de experiencias que suponen las juventudes “(...) vale decir, diversas expresiones y significaciones del entramado complejo que surge en nuestras sociedades desde un grupo social y que se expresa de maneras múltiples y plurales" (Duarte Quapper, 2000, p.70). En tanto clasificación, construida social e históricamente, establece límites respecto de otros actores sociales. Manifiesta conflictos y diferentes significaciones emergentes de las desiguales relaciones de clase, género, étnico-raciales, territoriales, etc. Supone una forma de construir lo etario donde cada sociedad instaura su segmentación e institucionalización generando categorizaciones diferenciales, valorizando moralmente ciertas prácticas, o incluso, invisibilizándolas (Chaves, 2013; Skelton, 2013).

6 Los sentires hacen referencia a las “(...) particulares maneras de ser/estar/desear/tener que ponen en juego los sujetos en el fluir de sus interacciones cotidianas" (Cervio, 2012, p.9). 
Dicho procesamiento social de las edades (Chaves, 2013), en tanto relación social, expresa relaciones de poder que, a su vez, se encuentran inmersas en una división social del espacio urbano que también es producto de disputas y relaciones de poder (Segura, 2017). En ese marco, la figura de los jóvenes fuera del hogar y su circulación por el espacio público es visualizada como problemática. Así, por ejemplo, la presencia de los jóvenes varones de sectores populares es asociada a la violencia y a la peligrosidad, por lo que son objeto de control y persecución. Suelen ser expulsados de espacios centrales como los centros comerciales o parques, ya que su presencia resulta inconveniente. Mientras que las jóvenes mujeres se tornan mayormente destinatarias de protección y cuidado. Sin embargo, la presencia juvenil también se manifiesta cuestionando usos legítimos de los espacios públicos a través de expresiones artísticas y políticas (Gough y Franch, 2005; Gough, 2008; Evans, 2009; Chaves y Segura, 2015; Gentile, 2017; Bonvillani, 2017).

En Latinoamérica, la producción y reproducción de las desigualdades sociales tiene un estrecho vínculo con la producción de los espacios urbanos (Segura, 2017). Las transformaciones de las ciudades latinoamericanas tras los procesos de reformas neoliberales -como la apertura económica y la desregulación del uso del suelo-, así como la globalización, agudizaron las desigualdades en dichos contextos. Esto ha operado sobre el desplazamiento: las posibilidades de circular y trasladarse cotidianamente, así como apropiarse del espacio urbano y, por otro lado, en tanto expulsión material y simbólica residencial, laboral y comercial- de ciertos grupos sociales respecto de determinadas áreas o espacios públicos de la ciudad (Blanco, Bosoer y Apaolaza, 2015). Como destaca Ramiro Segura (2017), si bien en los últimos años hubo un aumento en los ingresos, el "patrón de urbanización excluyente" se ha mantenido, e incluso, profundizado. De ello, se desprenden no sólo desigualdades en la calidad y la ubicación de las viviendas, sino también segmentación del acceso a la salud, la educación, el trabajo, las formas de movilidad, que (re)producen tales desigualdades. Una característica de este 
208 "No me agrada viajar". Moverse en la ciudad como desafío cotidiano para jóvenes de barrios populares de Buenos Aires

proceso ha sido que la segregación centro-periferia se ha complejizado, dando lugar a un proceso de fragmentación a escalas menores, al interior de áreas y barrios, generando en consecuencia:

(...) una aglomeración [tendencialmente] expandida, difusa, discontinua, policéntrica, de dimensión regional que implica cambios fundamentales en la organización y en el sentido mismo de la vida urbana (De Mattos, 2010: 96) y que tiene impactos significativos en términos de desigualdades sociales y espaciales. (Segura, 2017, p.28)

En el estudio de las vinculaciones entre lo urbano y las juventudes, si bien existen investigaciones que las abordan, “(..) la relación entre los procesos de fragmentación socio-espacial y la experiencia juvenil de la ciudad permanece poco explorada" (Saraví, 2014, p.1, traducción propia). No obstante, se destacan autores que distinguen la experiencia del espacio, y fundamentalmente de lo urbano, como una dimensión central y constitutiva de la vida de niños y jóvenes, al considerar que sus experiencias varían entre sí y se diferencian de la de los adultos. Al observar las lógicas del espacio urbano y su conformación través de la interacción de estructuras y movilidades de objetos, información y personas- es posible advertir que los jóvenes construyen formas particulares de circular, de construir vínculos, de (re)crear lugares propios. Por ello, resultan agentes significativos para comprender la conformación de lo urbano desde sus (in)movilidades (Duarte, 2000; Evans, 2009; Skelton, 2013; Saraví, 2014; Chaves y Segura, 2015; Bastán y Paulín, 2016; Sheller y Urry, 2016).

Ante las transformaciones y complejidades de lo social, y las experiencias juveniles en particular, entre los abordajes sociológicos se han generado indagaciones tendientes a centrar la mirada tanto en los procesos estructurales, en las experiencias y reflexividades de los individuos, como en sus posibles vinculaciones. En ese sentido, la sociología de la individuación que desarrolla Danilo Martuccelli se propone "reconstruir el carácter específico 
de una sociedad histórica a escala de sus individuos":

Esto quiere decir que, si es relevante conocer cómo se producen los individuos al enfrentar los problemas y requerimientos cotidianos y ordinarios -proceso de individuación-, al final del camino, el objetivo es diseñar una cartografía particular, capaz de describir a la sociedad y sus principales problemas, condensándola en un conjunto de grandes pruebas estructurales (...) que se deben enfrentar y en cuyo enfrentamiento se producen los individuos. (Araujo y Martuccelli, 2012, p.15)

Las pruebas se construyen como herramientas analíticas que permiten dar cuenta de procesos estructurales sociales e históricos que los individuos se ven obligados a atravesar, pero no de manera uniforme ni preestablecida. $\mathrm{Su}$ dimensión narrativa alude al modo en que los actores perciben esos desafíos, así como el trabajo que ello demanda. Enfrentar dichas pruebas, no supone un individuo autosuficiente o soberano, sino uno fabricado en sociedad que precisa soportes sociales, externos a él, para afrontar los desafíos sociales: “(...) no es, pues, de la constitución del individuo de lo que se trata, sino de los procedimientos por los cuales éste llega a tenerse frente al mundo. $\mathrm{Y}$ esos medios no son otros que el conjunto de los elementos, materiales e inmateriales, que lo vinculan a su contexto" (Martuccelli, 2007, pp. 61-62). Las condiciones de la vida urbana sugieren diferencias en los modos de ser joven, que segmentan recorridos escolares y oportunidades laborales, el acceso a prácticas $\mathrm{y}$ recursos culturales, a partir de los cuales se plantean significaciones y sentidos asociados a jóvenes de diferentes sectores sociales. Frente a las mismas pruebas -en este caso, la de las movilidades urbanas- se producen desigualdades en los soportes - materiales y simbólicos-a los que se acceden o pueden ser movilizados, pero también, cuáles son aceptados y legitimados (Miranda, 2008; Infantino, 2011; Corica, 2012; Pérez, Deleo y Massi, 2013; Di Leo y Camarotti, 2015). 
210 "No me agrada viajar". Moverse en la ciudad como desafío cotidiano para jóvenes de barrios populares de Buenos Aires

Desplazarse cotidianamente no es resultado de una decisión racional y autónoma, ni de un simple cálculo costo-beneficio (Jensen et al., 2014). La movilidad responde a necesidades y deseos, así como a la posibilidad de los individuos de satisfacerlos a través de diferentes usos del territorio que responden a condiciones de accesibilidad y apropiación material y simbólica diversas (Gutiérrez, 2009). Al entender la movilidad cotidiana como: “(...) las relaciones sociales, la creación y el mantenimiento de diferentes tipos de vínculos con lugares y personas, que no necesariamente están próximas, como los miembros de la familia o parientes, amigos y compañeros, empleadores o compañeros de trabajo, instituciones y servicios, etc." (Manderscheid, 2013 en Jensen et al., 2014, p.364, traducción propia), es preciso considerar la disponibilidad de recursos y servicios que ofrecen las distintas áreas urbanas y las posibilidades y limitaciones de los individuos para utilizarlos.

Analizar las formas en que se (re)crean formas de circular diferenciales permite advertir la producción de políticas de movilidad (Cresswell, 2010) que, al mismo tiempo, se intersectan con las políticas sobre los cuerpos y emociones. Éstas instituyen y regulan modos de sentir, de ser, de moverse, manifestando desigualdades en la disponibilidad de los cuerpos y las energías, la conformación de proximidades y distancias según las distintas posiciones sociales, pero también expresan resistencias y fugas, posibilidades de agencias y creatividad de los individuos frente a aquéllas (Scribano, 2011; 2012). En este trabajo, en vinculación con las herramientas conceptuales de la sociología de la individuación, se propone interrogar, asimismo, acerca de las posibilidades de pensar la movilidad cotidiana como una prueba, un desafío estructural al que todos se ven obligados a enfrentar y que representa un trabajo particular para los individuos en la búsqueda y movilización de soportes para moverse, permanecer, adaptarse, apropiarse del espacio urbano, así como los que se generan al circular cotidianamente. En el siguiente apartado se analizan los desplazamientos cotidianos de los jóvenes entrevistados a partir de tres dimensiones que resultaron significativas en sus relatos: a) obligaciones y 
opciones cotidianas, b) moverse intra/entre barrios, c) mediaciones en las movilidades cotidianas.

\section{JUVENTUDES Y MOVILIDADES COTIDIANAS URBANAS: UNA PROPUESTA ANALÍTICA}

\section{1 "PARA BIEN, ESTOY BASTANTE OCUPADO". OBLIGACIONES Y OPCIONES COTIDIANAS.}

Concurrir a la escuela en el centro de la ciudad desde un barrio del GBA. Asistir a un taller de acrobacia todos los sábados a la tarde, trasladándose desde la villa, en el límite con el GBA al centro de la ciudad. Estudiar, realizar diariamente tareas domésticas y cuidar de los hijos en una vivienda de un barrio del sur del GBA. Viajar en colectivo ${ }^{7}$ desde una villa, en los márgenes de un barrio de alto poder adquisitivo, hasta un skatepark ${ }^{8}$ en un área céntrica para juntarse a patinar y rapear con amigos. En su cotidianeidad, los entrevistados desarrollan actividades que articulan responsabilidades, rutinas y placeres. Aquí lo cotidiano refiere a:

(...) los encadenamientos y conjuntos de prácticas de los actores, como un tejido de redes en el espacio -más o menos densas- que tiene tanto aspectos objetivos como subjetivos, y pluralidades de sentido que se pueden condesar en elementos espaciales. Así como un tiempo, el de las 24 hrs. del día, que es cíclico y repetitivo, pero que está inserto en el tiempo histórico y en relación con el espacio-tiempo social. (González y

\footnotetext{
${ }^{7}$ Colectivo se designa cotidianamente al vehículo de transporte público interurbano, que tiene las características de un ómnibus o autobús.

8 "Un skatepark es básicamente un sitio preparado especial y artificialmente para la práctica del skate" (Saraví, 2012, p.35).
} 
212 "No me agrada viajar". Moverse en la ciudad como desafío cotidiano para jóvenes de barrios populares de Buenos Aires

Reyes, 2017, p.150)

A partir de este modo de comprender lo cotidiano resulta posible considerar que la finalidad que tienen los desplazamientos cotidianos, más que los lugares, son las actividades, los bienes y servicios que se sitúan en esos lugares. Es decir, son éstos los que movilizan a las personas a circular por diferentes áreas urbanas en pos de satisfacer sus necesidades y deseos (Gutiérrez, 2012). Tales prácticas conllevan, asimismo, el cumplimiento de obligaciones y responsabilidades. Habitualmente, concurrir al trabajo o a estudiar se ha vinculado con actividades que presentan menor flexibilidad en términos de ubicación y horarios a cumplir. Sin embargo, la circulación que ello supone, también habilita permanecer en lugares elegidos, desarrollar prácticas deseadas, viajar para participar de otras actividades, pudiendo elegir los momentos para llevarlas a cabo. Ello supone distintos requerimientos y necesidades según diferentes condiciones de género, edad, étnico-raciales socioeconómicas, etc. (Blanco, Apaolaza, Bosoer y González, 2014a).

Una lectura hacia las actividades cotidianas de los entrevistados podría suponer que, por ejemplo, aquellas prácticas realizadas en instituciones educativas o laborales implican obligaciones y responsabilidades que otras asociadas a lo recreativo no requerirían en términos de desplazamientos espacio-temporales. Sin embargo, lo relatado por los jóvenes expone la diversidad de significaciones que trasladarse para participar en cada espacio plantea para ellos. Aun cuando todos residen en barrios populares del AMBA, sus actividades no necesariamente se despliegan en estos barrios. Esto habilita a interrogar las posibilidades de elección de las actividades a desarrollar y en qué lugares, aunque ello no implica que dichas condiciones sean cuestionadas por los entrevistados. En el relato de Juan, uno de los entrevistados, se destacaba:

Después del laburo... salgo antes y me voy (...) Me tomo el subte ${ }^{9} \mathrm{y}$

\footnotetext{
${ }^{9}$ Subte es la denominación cotidiana de un tipo de transporte público de la CABA, con forma de ferrocarril subterráneo.
} 
llego al toque [rápido] Hago combinación y llego... Y es como que ya voy ahí, siempre fui ahí. Si no acá tenés, pero no me gusta porque no tiene... (...) Hace rato que patino ahí, me gusta patinar ahí (...) Ya conoces la plaza, ya sabes lo que hay, lo que podés hacer y no sé, no sé... está más buena. Igual si hubiese un skater acá cerca iría acá nomás. [Juan, bachillerato, CABA]

El joven trabaja en un programa estatal y concurre a un bachillerato popular. Vive en uno de los barrios de la CABA constituido a partir de un asentamiento, paradójicamente, ubicado en los márgenes de una de las áreas de mayor poder adquisitivo del centro de la ciudad, Puerto Madero. Luego de cumplir con el horario laboral, elige viajar hasta un skatepark, que se encuentra en otro barrio del centro, para patinar y rapear, pero fundamentalmente para encontrarse con amigos, prácticas y relaciones que actúan como soportes significativos en su vida cotidiana. Ello requiere el traslado en un colectivo que lo lleva directo o a través de la combinación de dos subtes. En su investigación, Guido Bastan y Horacio Paulín (2016), analizan las construcciones identitarias de jóvenes de barrios populares urbanos de la provincia de Córdoba, Argentina. Los autores señalan que cuando comienzan a viajar solos al 'centro' de la ciudad se trasladan para buscar trabajo, realizar trámites o para encontrar espacios de sociabilidad juvenil. Éstos últimos, por ejemplo los grandes centros comerciales, son lugares por los cuales los jóvenes desean circular, pero que resultan inexistentes en sus barrios, por lo que la necesidad de viajar resulta casi inevitable. En el caso de Juan, el traslado que realiza cotidianamente hacia el skatepark -de la villa al centro- no resulta problemático. Sin embargo, menciona que de existir un espacio como ese en el lugar donde vive, probablemente concurriría a éste, en lugar de trasladarse a otro barrio.

Ante la pregunta por el modo en que llegaba desde su casa en el barrio de Banfield del GBA, hasta el circo en el sur de la CABA, Víctor hacía un paralelo con el viaje que ha realizado todos los días desde su barrio hasta la 
214 "No me agrada viajar". Moverse en la ciudad como desafío cotidiano para jóvenes de barrios populares de Buenos Aires

escuela a la que concurre en el centro de la ciudad:

(...) Difícil no se me hace porque yo estudiaba en el Colegio, acá a unas cuadras, el [nombre de la escuela] que era acá nomás... O sea, es el recorrido que hice durante 6 años. [Claro, ¿qué tomás, un colectivo?] Un colectivo hasta la Estación. De la Estación un tren hasta Constitución y de Constitución me tomo el colectivo. [Víctor, circo, GBA]

Víctor ha concurrido a una escuela técnica reconocida del centro de la CABA. Cada día supuso para él viajar alrededor de una hora u hora y media desde su casa, articulando tres medios de transporte: colectivo-tren-colectivo. La elección de escuelas en zonas céntricas de la ciudad resultó un emergente en otro trabajo (Tapia, 2016) vinculado con la decisión de adultos de seleccionar instituciones que, asociadas a su ubicación, se presentan con mayor prestigio que aquéllas situadas en los barrios populares. Estas últimas, por el contrario, se presentaron vinculadas a sentidos negativos y a problemáticas como el embarazo temprano, el uso de drogas y la deserción, que los adultos generalmente padres y madres-, desean evitar para sus hijos para quienes proyectan futuros prósperos a partir de una buena educación. Esos futuros tienen la posibilidad de alcanzarse, siempre y cuando se establezcan distancias, materiales y simbólicas, entre los jóvenes y las influencias negativas que los barrios populares pueden ocasionar.

Ahora bien, más allá del imaginario de padres y madres, estas decisiones también requieren ser relacionadas con la distribución en el territorio, real y diversa, de la calidad de los servicios y oportunidades en cada área. Como señala Gutiérrez (2012), aunque sea menos perceptible que dar cuenta de la inexistencia de un servicio, por ejemplo de salud, educación o empleo en un determinado sector urbano, observar su calidad también resulta sustantivo para dar cuenta de las necesidades de movilidad requerida para los distintos grupos sociales. En ese sentido, debe destacarse la escasez de instituciones públicas, por ejemplo escolares y de salud, de calidad en estos barrios (Segura, 2017). 
Cabe destacar que en el relato de Víctor no se plantea un cuestionamiento a la necesidad de concurrir a un establecimiento alejado de su lugar de residencia, ni al desplazamiento diario que éste supone. Por el contrario, el camino recorrido durante seis años es advertido como una posibilidad de aprendizaje, una especie de habilidad que facilita -a partir del conocimiento adquirido en la práctica del viaje a la escuela- la posibilidad de llegar sin mayores problemas a una nueva institución, aun cuando la distancia geográfica sea significativa. Franz Buhr (2017) en un estudio acerca del aprendizaje e integración en el uso de la ciudad por parte de los migrantes en España, advierte que dicho uso requiere habilidades y conocimientos para poder tomar decisiones que permiten conocer y usar la ciudad como los itinerarios y horarios de los transportes, el tener noción de los lugares, rutas y las opciones disponibles para ser usadas. Este conocimiento urbano “(...) no es adquirido, sino producido en la relación persona-entorno y siempre condensa una conexión especifica entre urbanidades corporizadas y territorios urbanos particulares" (Buhr, 2017, p.15).

Ramona, otra entrevistada, es la encargada de realizar las tareas domésticas en su casa, ya sea cocinar la comida para su marido y sus hijos, como de encargarse de la limpieza del hogar o llevar y traer a sus hijos al jardín, ubicado en el mismo barrio en el que reside en el Conurbano bonaerense. Al relatar su rutina diaria, ella describía:

Me levanto temprano, a las cinco de la mañana, a cocinarle a él [pareja]. Se va a trabajar, depende adonde se tenga que ir. A las siete, ya mis hijos se levantan a desayunar, a cambiarse para el jardín. Los llevo al jardín, llego a mi casa, me pongo a limpiar, me quedo con mi nena chica allá y a las doce ya... ya están en mi casa, ya comen, todo. Se acuestan a dormir siesta y a la tarde ya llega mi marido. Ya está todo cocinado, limpio. Se queda con los chicos y yo vengo al bachi. Cuando yo ya vengo, ellos ya están todos preparados y ya se acuestan temprano, como 
216 "No me agrada viajar". Moverse en la ciudad como desafío cotidiano para jóvenes de barrios populares de Buenos Aires

se levantan temprano, ya están todos durmiendo para cuando llego. [Ramona, bachillerato, GBA]

Su marido "se va" a trabajar, ella trabaja y organiza su casa, moviéndose en su hogar para que otras movilidades, como la de su pareja o el llevar a sus hijos al jardín, sucedan en el momento indicado. Como señalan diversas investigaciones, las movilidades presentan patrones diferenciales por género que exceden la distinción entre lo público y lo privado (Gutiérrez, 2009; Chaves et al., 2017; Jirón y Zunino Singh, 2017; Jirón, 2017). Si bien las vidas cotidianas de las mujeres se han asociado al ámbito doméstico, “(...) la experiencia cotidiana de las mujeres no se circunscribe necesariamente al ámbito de la casa, ni el de los varones al ámbito de la calle" (Chaves et al., 2017, p.45). En el caso de Ramona, gran parte de las responsabilidades del hogar dependen de ella. Sin embargo, incluso en un horario nocturno, suspende dichas tareas -que son, en cambio, realizadas por su pareja- para asistir al bachillerato donde estudia para terminar el secundario. Por estar dentro del barrio y poder llegar caminando, ha podido sostener dicha actividad por tres años. Actividad que realizaba en una escuela secundaria de otro barrio cercano y que había abandonado tras su primer embarazo.

La cotidianeidad de los jóvenes de barrios populares puede resultar muy diversa, aun cuando suponga condiciones de vida similar en términos económicos, sociales o de género (Chaves y Segura, 2015). Para Tincho y Jorge, las actividades que realizan diariamente requieren rutinas donde el espacio-tiempo expresa ritmos diferentes:

Para bien estoy bastante ocupado. Lunes a las seis levantarme, desayunar, comer, prepararme para ir a hacer la pasantía. Pasantía de ocho a doce, de doce a una comer y trasladarme para la escuela, de una a cinco la escuela, todo teoría y lo que me sobra, dependiendo... Los martes y jueves voy al curso de [capacitación para el trabajo en el circo] de seis a nueve. No, de seis a ocho, martes y jueves. Lunes, miércoles y 
viernes y sábado a la tarde, de seis a nueve, voy al gimnasio. Y bueno, sábado vengo para acá [el circo] (...) El único día libre es el domingo. [Tincho, circo, CABA]

Me levanto y vengo al COP hasta el mediodía. Al mediodía, a mi casa y de mi casa... no sé, me pongo a ver la tele, algo... Y ya... cuando... es 4:30, me toca ir a buscar a mis hermanas. A las $6: 10,6: 15$ estoy en mi casa otra vez y me pongo, no sé, a hacer algo... hasta la hora de dormir. [Jorge, COP, CABA]

La opción por no moverse, elegir descansar, capacitarse o realizar una actividad artística no se presentan de la misma manera para estos dos jóvenes varones. En el primer caso, el único momento libre de actividades y desplazamientos, llega el último día de la semana. En el segundo caso, en cambio, luego de realizar una práctica de formación por la mañana y de encargarse del traslado de sus hermanas desde la escuela a la casa en un momento de la tarde, en el día de Jorge no se advierten oportunidades o alternativas al "ver algo en la tele" o "hacer algo...", fuera o dentro del hogar. Sin embargo, más adelante en su entrevista, dirá que tiene una actividad que le gusta: "manejar", “(...) la verdad que me relajo bastante poder manejar. Cuando estoy nervioso, salgo con el auto y me tranquilizo un montón...". Si bien no lo realiza muy a menudo, es importante destacar que una práctica de movilidad, fuera de su casa en la que pasa la mayor parte de su tiempo, es una estrategia que le permite relajarse y sentirse "cómodo" al recorrer la ciudad.

\section{2 "EN MI BARRIO HAGO TODO". MOVERSE INTRA/ENTRE BARRIOS.}

En los emergentes de las entrevistas fue posible registrar que desplazarse por diferentes áreas del AMBA genera distintas percepciones y sentires de los jóvenes acerca de los barrios por los que transitan. En particular, 
218 "No me agrada viajar". Moverse en la ciudad como desafío cotidiano para jóvenes de barrios populares de Buenos Aires

tensiones respecto de la peligrosidad o seguridad que provoca transitar en el espacio urbano: para algunos, asociada a las violencias dentro del barrio y para otros, a la falta de seguridad que genera lo no conocido, por ejemplo, al viajar por un barrio diferente al propio. Para Carla, la inseguridad se proyecta fuera del barrio. Ella prefiere circular y realizar la mayor cantidad de actividades dentro de éste:

No, no me siento segura, no me siento segura (...) no sé, tal vez es una sugestión que se genera, por ejemplo, por los rumores que se corren, no sé, esto de las chicas que secuestran y no sé, que te roban, uno sale medio perseguido (...) me da miedo... (...) Permanezco en el barrio (...) Todo el día en el taller y no salgo. Y en sí si tengo que hacer otra cosa, hacer algo siempre trato de estar, de poder hacerlo en el barrio [Carla, bachillerato, GBA]

Gran parte de las investigaciones analizan movilidades actuales y pasadas, siendo menos observada la potencialidad, es decir, las posibilidades y limitaciones que experimentan los individuos para maniobrar en sus vidas cotidianas y poner en práctica su capacidad de ser móviles, así como las consecuencias sociales, económicas y simbólicas que ello produce (Kaufmann et al., 2004). En el contexto australiano, un estudio comparativo indaga la conformación de identidades y sociabilidades juveniles a partir de las (in)movilidades urbanas. Al analizar las experiencias de jóvenes "isleños" zona más empobrecida- se advierte que las posibilidades de salir de sus barrios para transitar por la ciudad - espacio de personas "blancas"- genera en ellos la sensación de sentirse fuera de lugar. En consecuencia, los jóvenes auto limitan sus posibles traslados hacia la ciudad por el temor a no ser bienvenidos dada su condición socioeconómica y étnica, que no encajaría con ese modelo de ciudad (Skelton, 2013).

En el relato de Carla, sus potenciales desplazamientos se ven limitados por el "miedo" que genera lo que se construye como el "afuera" del barrio. 
Miedo sustentado, fundamentalmente, en sensaciones creadas a partir de los "rumores que corren". Como consecuencia, sea para trabajar, realizar trámites o estudiar, ella encuentra en el barrio la opción más segura para llevar a cabo esas actividades. Gabriel Kessler (2015, p.48) ha destacado, siguiendo a Rossana Reguillo, el modo en que el miedo, “(...) es una experiencia individualmente experimentada, socialmente construida y culturalmente compartida. Como forma de respuesta, se trata del plano de lo individual; sin embargo, la sociedad es la que construye las nociones de riesgo, amenaza y peligro (...)". Si bien ella no lo menciona, si se consideran estigmatizaciones hacia los jóvenes de barrios populares, quienes son juzgados como peligrosos y amenazantes, ese miedo de Carla puede estar vinculado también a no ser aceptada o reconocida como una integrante legítima de la ciudad al circular por ésta.

En el caso de Mariana, que ha vivido en una villa en el sur de la CABA, en límite con el GBA, desde su niñez, aun cuando transitar por este barrio, le resulta familiar y conocido, la distinción entre un adentro y un afuera supone para ella una diferenciación en los vínculos que genera:

Hay mucha gente que por ahí me conoce de chiquita que yo no conozco. O sea, no, no tengo mucha relación con la gente que vive dentro del barrio. Sólo con algunas, pero por ahí... capaz yo voy caminando con mi abuela o con mi mamá y todos las saludan y a mí también. Me conocen de chiquita, pero por ahí no conozco a esa persona y no... no me relaciono mucho con la gente dentro del barrio. Prefiero relacionarme con gente de afuera [Mariana, circo, CABA]

En el barrio la conocen, vive su familia, pero las relaciones de amistad deseada se buscan al desplazarse por fuera del barrio, por ejemplo, aquéllas generadas en la escuela pública a la que concurre en un barrio de clase media más cercano al centro de la ciudad. Como señalan algunos estudios, la distinción entre el afuera/adentro ha sido señalada como una manera de conformar simbólicamente los límites entre barrios y contraponer sentidos - 
220 "No me agrada viajar". Moverse en la ciudad como desafío cotidiano para jóvenes de barrios populares de Buenos Aires

como inseguridad/seguridad o peligrosidad/tranquilidad- asociados a las características de cada barrio y la población que los habita. Vinculación que resulta de una construcción producida por diferentes actores como el Estado, el mercado, los medios de comunicación y la misma población residente en estos territorios (Cravino, 2009).

La asociación entre movilidad cotidiana, género y edad puede visibilizar experiencias diferentes y desiguales para los y las jóvenes. Gutiérrez y Reyes (2017), en ese sentido, señalan que: “(...) las mujeres son un grupo social sensible a factores espacio-temporales, como la distancia y las condiciones del viaje, la disponibilidad y horarios del transporte público, entre otros” (p. 149). En un estudio realizado en Santiago de Chile, al analizar las movilidades y barreras de accesibilidad urbana cotidianas, se identifica el modo en que dos mujeres -de bajos y altos ingresos- desarrollan diferentes tácticas y estrategias. Se destaca, en la de bajos ingresos, la necesidad de resolver la organización del cuidado de su hijo siendo madre soltera, la elección de un tipo de trabajo con horarios convenientes, la búsqueda de transportes económicos, cómodos y prácticos o la opción de circuitos más seguros en su recorrido cotidiano. En el caso de las jóvenes aquí entrevistadas fueron ellas, especialmente, las que destacaron la necesidad de modificar recorridos o cambiar de lugares a los que se concurre para evitar circular en horarios o zonas que se consideran peligrosas e inseguras, ya sea dentro o fuera de sus barrios. Sus traslados se realizan en transporte público - sobre todo en colectivo, ya que los subtes no llegan a estos barrios- y en menor medida, dado su mayor costo, en remis, ${ }^{10}$ mayormente para salidas nocturnas del fin de semana o para el traslado de niños. Ante tales situaciones, no obstante, las entrevistadas encuentran estrategias ${ }^{11}$ y soportes

\footnotetext{
${ }^{10}$ Remis es un medio de transporte informal de Argentina que, con un funcionamiento similar al del taxi, emplea un automóvil con conductor para realizar traslados con una tarifa fija por trayecto realizado.

${ }^{11}$ La noción de estrategia supone decisiones en las que intervienen múltiples factores. Aunque las decisiones de movilidad se encuentran vinculadas con las estructuras de opciones, y aún con limitada posibilidad de acción, este término permite reconocer
} 
para desplazarse y mantener sus actividades. En sus entrevistas, dos de ellas relataban:

Como para entrar a mi casa, hay dos entradas. La parte de una canchita y la entrada general. Y por lo general si es de noche o si salgo sola entro por la entrada general que es donde se moviliza más gente y como suelen haber tantos problemas... Y si salgo con alguien, salgo por el otro lado, que me queda más cerca de la parada, pero... por lo general no ando sola por ahí porque es donde se juntan más los chicos que fuman. Entonces, para evitar ciertas cosas es preferible no estar sola. [Mariana, circo, CABA]

(...) salía muy tarde allá porque yo entraba a la noche ahí, yo trabajaba a la mañana, y a la tarde me iba ahí y ya salía muy tarde porque entraba turno noche (...) En el colectivo viajaba. El colectivo tardaba mucho y ya llegaba muy tarde y era muy peligroso, también (...) Y de vez en cuando, sí, mi mamá me buscaba, pero cuando podía... (...) Y acá, voy caminando a mi casa porque está cerca acá... me queda más cerca, no tengo viaje. Y bueno, ya no me complica más ni el colectivo que me lleve tarde, ni nada. Puedo venir sola. Caminando. [Fatu, bachillerato, $\mathrm{CABA}]$

En el caso de Mariana, su conocimiento del barrio puede construir un espacio con diferentes entradas, los recorridos que cada una supone y los modos de transitar por éstos. Ya sea uno u otro camino, la presencia de otros -que la acompañen o que se desplacen por allí- actúa como un soporte útil y necesario para las salidas y entradas. Para Fatu, la presencia de la madre es observada

una parte de decisión de los individuos, su capacidad de agencia, y habilita un espectro de posibilidades. Ello supone además contar con soportes para que la estrategia sea posible. En ese sentido, se consideran las estrategias individuales en interacción con los condicionantes estructurales, por lo que aún en contextos similares, las experiencias y cursos de acción puede ser muy diversos (Bonvalet y Dureau, 2002; Di Virgilio y Gil y de Anso, 2012). 
222 "No me agrada viajar". Moverse en la ciudad como desafío cotidiano para jóvenes de barrios populares de Buenos Aires

como un soporte facilitador para transitar entre la parada del colectivo y su casa. Sin embargo, dado el horario de cursada de la escuela secundaria a la que asistía y el viaje que implicaba, tanto por la espera como la distancia, no pudo concurrir más a dicha institución. La posibilidad de seguir estudiando, evitando dicho viaje, se produce al asistir al bachillerato popular del barrio donde reside, donde no hay otras escuelas secundarias.

Reconocer las visiones subjetivas acerca de los espacios urbanos y los modos de moverse en y a partir de ellos, resultan de significaciones que son múltiples y dinámicas, que construyen experiencias muy diversas aun estando situadas en un mismo territorio. Atender a las percepciones y sentires que generan tales territorios y las movilidades que los conforman, habilitan, asimismo, una mayor comprensión no sólo de los desplazamientos efectuados, sino también aquellos que son evitados, deseados, elegidos e incluso, no realizados (Gutiérrez, 2012).

\section{4. "No ME AGRADA VIAJAR". Mediaciones EN LAS MOVILIDAdES COTIDIANAS.}

Estar cómodo, tranquilo. Emociones opuestas a las que el uso de medios de transporte públicos, -en particular, los colectivos- generan en los jóvenes entrevistados. En sus relatos, los desplazamientos son asociados con sensaciones de malestar, incomodidad, cansancio. Gabriel Kessler (2015) en su análisis del entramado de representaciones, acciones y emociones que engloba bajo la noción de "sentimiento de inseguridad" (2015), siguiendo la crítica de Arlie Hochschild (1983) a la concepción organicista de las emociones, señala que su constitución no refiere a “(...) procesos individuales internos, en los que la introspección y la relación con los otros serían posteriores y exteriores a la conformación de la emoción. Antes bien, plantea que las interacciones 
intervienen en la definición misma de la emoción (...)”(pp. 43-44).

Las distancias desde los lugares de residencia y los espacios en los que participan los jóvenes requieren, al menos, la utilización de un colectivo para trasladarse cotidianamente entre el hogar y la escuela, el bachillerato y el trabajo, el trabajo y el circo, el jardín de infantes de los hijos o hermanos y el hogar, la plaza para encontrarse con amigos y el trabajo:

Mi papá sigue viviendo en Quilmes, mi abuela también. Lo que pasa es que tal vez, a mi Quilmes me gusta, pero tal vez el viaje, el colectivo es un embole [fastidioso], eso es lo que me molesta. Y tener que viajar y eso no me gusta para nada. [Carla, bachillerato, GBA]

Mucho no me gusta viajar, no soy muy amante de estar todo el tiempo en el colectivo, no, no me gusta. Me siento incomodo, no sé, raro... No sé, no, mucho no me gusta. Y si no tengo el celular con los auriculares, menos, menos... no. [Jorge, COP, CABA]

Los viajes en transporte público representan prácticas que se desean evitar. Viajar es "un embole" [molesto], "raro", "incómodo". Aun cuando no se trate de distancias muy grandes, suponen un uso considerable de tiempo diario. Sin embargo, los jóvenes frente a ello utilizan estrategias para atravesar estos espacios-tiempo de manera más agradable. Así, por ejemplo, los auriculares para escuchar música pueden convertirse en objetos que permiten 'soportar' mejor los viajes. Otra de las prácticas que es apreciada por los jóvenes es la caminata. Prefieren bajar en paradas previas a las que les corresponden para disfrutar de ese tramo del camino que el viaje en colectivo parece obstaculizar. Víctor y Tincho, ambos participantes de talleres del circo destacaban en sus relatos:

Plazas, acá, donde hago taekwondo... Ahí me siento mucho más tranquilo, me siento más cómodo [¿Qué te gusta de las plazas por ejemplo?] La tranquilidad. En el anterior lugar donde hacíamos yo me 
224 "No me agrada viajar". Moverse en la ciudad como desafío cotidiano para jóvenes de barrios populares de Buenos Aires

bajaba mucho antes para caminar a la plaza nada más. Me podría haber bajado dos cuadras y caminar dos cuadras, pero yo me bajaba como cinco y caminaba toda la plaza. Era la tranquilidad (...) Acá no se puede. Me bajo a una cuadra [¿Y no te podés bajar antes o no hay nada atractivo antes?] Es que la zona no te da mucha confianza. [Víctor, circo, CABA]

[El circo] Tenía una sede muy cerca de mi casa y lamentablemente ya no es, pero igual sigo participando (...) me quedaba 9 cuadras, 10. Caminaba tranquilamente [¿Y para venir para acá como haces?] Colectivo, sí. [Tincho, circo, CABA]

Frente a los ritmos impuestos por la vida urbana moderna que supone velocidad en la circulación de personas, de objetos y de información (Cresswell, 2010), los decires de los jóvenes ponen de manifiesto necesidades de "tranquilidad", de tener momentos que les permitan llevar su propio ritmo, de las maneras deseadas. Una de estas formas deviene del "caminar" por lugares elegidos, como una plaza, un espacio verde de la ciudad. En este usar el espacio, caminando, éste adquiere una dimensión corporal, viva, que supone colores, ritmos y formas de moverse, que se articulan con la dimensión material de los edificios, los medios de transporte, las calles (Jensen et al., 2014; Buhr, 2017).

Sin embargo, el hecho de que la institución a la que concurren se haya trasladado a un nuevo edificio modifica los recorridos de los jóvenes. Para ellos, esto genera un mayor peligro que dificulta esa forma de moverse tan deseada. En un estudio realizado en Lusaka, África, Mónica Gough (2008) analiza las movilidades cotidianas de jóvenes de sectores populares. En su trabajo registra el modo en que caminar es el medio de transporte para aquéllos con menores ingresos. En el caso de los jóvenes, éstos suelen caminar para concurrir a sus escuelas -en general, en compañía de otros jóvenes-, debiendo recorrer hasta más de $6 \mathrm{~km}$. diariamente. Sólo pocos pueden afrontar los costos 
de los ómnibus e incluso, muchos no cuentan con alimentos suficientes en sus hogares, por lo que suelen caminar sin haber desayunado previamente. En el AMBA, un análisis de las movilidades cotidianas urbanas según ingresos (Blanco, Bosoer y Apaolaza, 2014b), registra una diferenciación por condición socioeconómica en la apropiación de la ciudad y sus redes de servicios de transporte. En el caso de los sectores más empobrecidos su movilidad se asocia al uso del transporte público y a la caminata como forma de desplazamiento más utilizado. Los medios de transporte urbano que utilizan se caracterizan por su deficiencia, incumplimiento de horarios, tiempos de viaje elevados, confort limitado, alta congestión. En el deseo de "tranquilidad" que buscan los entrevistados es posible advertir una forma de "soportar" la necesidad de desplazarse en tales contextos, donde la elección es entre caminar o utilizar estos transportes al no contar con automóvil o bicicleta, como sucede en estos casos.

Adrián Scribano (2010, p.181) señala que en nuestras sociedades ciertas prácticas se hacen cuerpo como mecanismos de soportabilidad social que sostienen un orden social y económico, evitan el conflicto y ponen de manifiesto corporalidades y sensibilidades que llevan a tolerar las injusticias y desigualdades de manera naturalizada, al ser vividas como experiencias individuales y privadas. Así, por ejemplo, la 'espera' en ámbitos institucionales requiere aprender a tolerar hasta ser atendido, tener una postura correcta, estar atentos, sin quejarse y con 'paciencia'. No obstante, también es posible resistir, resignificar, tales condiciones. Así, caminar puede resultar una necesidad y, a su vez, una fuga (Scribano, 2012) de los límites que impone dicho contexto, pudiendo ser vivenciado como una forma de relajarse frente a las dificultades que deben atravesarse al utilizar el transporte público que, en el caso de los entrevistados, adquiere a su vez una forma de experimentar un movimiento propio y vital que se vuelve un soporte para ellos. 
226 "No me agrada viajar". Moverse en la ciudad como desafío cotidiano para jóvenes de barrios populares de Buenos Aires

\section{REFLEXIONES FINALES}

De la propuesta analítica, en la que confluyeron aportes de la sociología de la individuación y de los estudios sobre movilidades espaciales se advirtió que en contextos de desigualdad, fragmentación urbana y segmentación de oportunidades, las (in)movilidades cotidianas urbanas se vuelven pruebas para los jóvenes de barrios populares del AMBA. Enfrentar estos retos cotidianos requiere un trabajo significativo para ellos, para lo cual movilizan diferentes soportes y estrategias que les permiten afrontarlos.

Por un lado, su presencia en la ciudad ha sido observada como potencial peligro o ha sido desplazada de ciertos espacios, donde su presencia no resulta bienvenida. Por otra parte, sin considerar los soportes materiales y simbólicos que ello requiere, son interpelados a desarrollar prácticas como el estudio y el trabajo, que son más valorizadas cuando son realizadas fuera de los barrios en los que éstos residen. En consecuencia, los jóvenes deben trasladarse cotidianamente para asistir a escuelas, trabajos -que suponen mayor calidad y prestigio- y hasta para participar de espacios de sociabilidad y recreación. Sin embargo, aun compartiendo condiciones territoriales, etarias, de clase o de género, sus experiencias expresan diversidades en el modo en que perciben y experimentan sus (in)movilidades cotidianas.

Para las jóvenes, por ejemplo, ser madre joven puede presentar la dificultad de seguir estudiando en las escuelas que no contemplan las posibilidades o limitaciones de sus movilidades y temporalidades. Por otro lado, circular dentro/fuera de sus barrios genera peligros y temores que obstaculizan la elección de horarios, recorridos o los medios de transporte utilizados. Frente a ellas, las jóvenes ponen en juego estrategias individuales o movilizan soportes como la concurrencia a los bachilleratos populares -con horarios y ubicaciones más accesibles-, la búsqueda de la compañía de otros o la elección de ciertos recorridos para circular por la ciudad y sostener sus 
actividades. En el caso de los varones, la organización semanal para algunos se completa con una grilla de actividades como el estudio, el trabajo, espacios de sociabilidad en un skateboard o el circo. No obstante, se evidencian otras experiencias de varones que -más cercana a la de las jóvenes que son madresrealizan tareas de cuidado de otros o permanecen en la vivienda durante gran parte del día, siendo sus desplazamientos diarios más asociados y reducidos a lo doméstico. Así, si bien el género pone de manifiesto condiciones diferenciales, la articulación con la dimensión territorial y la fragmentación urbana permite vislumbrar que las limitaciones o posibilidades de los jóvenes para transitar por diferentes espacios, refiere también a los recursos disponibles en cada área, como la existencia de escuelas secundarias o diversidad de medios de transportes en estos barrios. Por ello, resulta relevante para el análisis de las movilidades cotidianas y su vínculo con los procesos de desigualdad -en términos económicos, sociales, culturales y simbólicos-, considerar tanto los desplazamientos que son elegidos y realizados, como también aquellos que son evitados, temidos o no son llevados a cabo.

Diferentes significaciones y sentires se generan al desplazarse por la ciudad y cumplir con requerimientos y actividades, pero también al realizar las actividades que son elegidas y deseadas, por lo que no son posibles de establecerse a priori en relación con lugares o formas de movilidad determinados, sino que se configuran en el proceso de aprendizaje del uso y apropiación del espacio urbano que cada joven ha realizado. Los jóvenes buscan contar con vínculos y lugares elegidos, placenteros, con ritmos propios. Así, incluso cuando requieran viajar largas distancias o por períodos extensos, ello no necesariamente es vivido como una dificultad, sino que también puede ser resignificado como espacio de elección, de aprendizaje, de apropiación y disfrute de la ciudad, y los modos de moverse en ella.

Los recursos con los que cuentan para ello, los obliga a utilizar medios de transportes públicos y realizar recorridos cuyos rasgos se asocian a la 
228 "No me agrada viajar". Moverse en la ciudad como desafío cotidiano para jóvenes de barrios populares de Buenos Aires

precariedad, a la congestión, la lentitud. Ello genera en los entrevistados sensaciones de cansancio, de molestia e incomodidad a los cuales sólo cabría adaptarse. Sin embargo, buscan 'fugarse' de estas condiciones encontrando soportes y estrategias que los ayudan a transitar estos espacios-tiempos de formas más placenteras que emergen en los mismos desplazamientos cotidianos: utilizar auriculares para escuchar música, optar por bajarse antes del colectivo para caminar o pasear por una plaza permiten desplazarse de formas más agradables y propias. Asimismo, el acceso a instituciones que generan oportunidades educativas, laborales y culturales -como es el caso de un circo, un centro de formación o los bachilleratos populares aquí estudiados-, resultan instituciones productoras $\mathrm{y}$ producidas en recorridos $\mathrm{y}$ temporalidades diferentes a otras como la escuela o la familia que se tornan soportes de relevancia para los jóvenes.

El análisis propuesto ha buscado constituirse, de este modo, en un aporte para reflexionar acerca del modo en que las intervenciones institucionales y políticas orientadas a jóvenes pueden considerar sus cotidianeidades, considerando sus posibilidades y limitaciones en el acceso, las potencialidades de sus movilidades para llegar a las instituciones, así como también las dificultades que enfrentan para lograrlo.

\section{REFERENCIAS}

Araujo, Kathya y Martuccelli, Danilo (2012). Desafios comunes. Retratos de la sociedad chilena y sus individuos. Santiago de Chile: LOM.

Bastán, Gastón y Paulín, Horacio (2016). Identidades juveniles en escenarios de periferización urbana. Una aproximación biográfica. Quaderns de Psicologia, 18(1), pp. 35-52.

Blanco, Jorge; Apaolaza, Ricardo; Bosoer, Lucía y González, Ariel (2015). Movilidades, desplazamientos y territorios. Algunos aportes para el debate sobre la gentrificación. Working Paper Series Contested Cities.

Blanco, Jorge; Bosoer, Lucía y Apaolaza, Ricardo (2014a). Gentrificación, movilidad y transporte: aproximaciones conceptuales y ejes de 
indagación. Revista de Geografía Norte Grande (58), pp. 41-53.

Blanco, Jorge; Bosoer, Lucía y Apaolaza, Ricardo (2014b). Movilidad, apropiación y uso del territorio: una aproximación a partir del caso de Buenos Aires. Scripta Nova Revista electrónica de geografía y ciencias sociales. 18(493).

Bonvalet, Catherine y Dureau, Françoise (2002). Los modos de habitar: decisiones condicionadas. En F. Dureau, V. Dupont, E. Lelièvre, JP Lévy y T. Lulle (coord.), Metrópolis en movimiento: una comparación internacional, Bogotá: Alfaomega., pp. 69-88.

Bonvillani, Andrea (2017). Emocionalidad y espacio público: detenciones arbitrarias de jóvenes de sectores populares de Córdoba (Argentina). Cuaderno urbano, 23(23), pp. 1-10.

Buhr, Franz (2017). Using the city: migrant spatial integration as urban practice. Journal of ethnic and Migration Studies, pp. 1-14.

Cervio, Ana (2012) (Comp.). Las tramas del sentir: ensayos desde una sociología de los cuerpos y las emociones. Buenos Aires: Estudios Sociológicos Editora.

Chaves, Mariana; Segura, Ramiro; Speroni, Mariana; Cingolani, Josefina (2017). Interdependencias múltiples y asimetrías entre géneros en experiencias de movilidad cotidiana en el corredor sur de la Región Metropolitana de Buenos Aires (Argentina). Revista Transporte $y$ Territorio, pp. 41-67.

Chaves, Mariana y Segura, Ramiro (eds.) (2015). Hacerse un lugar. Circuitos y trayectorias juveniles en ámbitos urbanos. Buenos Aires: Biblos.

Chaves, Mariana (2013). Culturas juveniles en la tapa del diario: tensiones entre el margen y el centro de la hoja. En M. Chaves y E. Fidalgo (comp.), Politicas de infancia y juventud. Producir sujetos y construir Estado. Buenos Aires: Espacio Editorial.

Corica, Agustina (2012). Las expectativas sobre el futuro educativo y laboral de jóvenes de la escuela secundaria. Última Década, 36, pp. 71-95.

Cravino, M. Cristina (2009). Vivir en la villa. Relatos, trayectorias y estrategias habitacionales. Buenos Aires: Universidad Nacional de General Sarmiento.

Cravino, Ma. Cristina; Duarte, Juan y Del Rio, Juan (2008). Magnitud y crecimiento de las villas y asentamientos en el AMBA en los últimos 25 años. Buenos Aires: ULACAV.

Cresswell, Tim (2010). Towards a Politics of Mobility. Environment and planning. D, Society and space. 28 (1), pp. 17-31.

Di Leo, Pablo y Camarotti, Ana C. (Dir.). (2015). Individuación y 
230 "No me agrada viajar". Moverse en la ciudad como desafío cotidiano para jóvenes de barrios populares de Buenos Aires

reconocimiento. Experiencias de jóvenes en la sociedad actual. Buenos Aires: Teseo.

Di Leo, Pablo y Camarotti, Ana C (eds.). (2013). 'Quiero escribir mi historia'. Vidas de jóvenes en barrios populares. Buenos Aires: Biblos.

Di Virgilio, M. Mercedes y Gil y De Anso, M. Laura (2012). Estrategias habitacionales de familias de sectores populares y medios residentes en el área metropolitana de Buenos Aires (Argentina). Revista de Estudios Sociales, pp. 158-170.

Duarte Quapper, Klaudio (2000). ¿Juventud o Juventudes?: Acerca de cómo mirar y remirar a las juventudes de nuestro continente. Última década, 8(13), pp. 59-77.

Evans, Bethan (2009). Geographies of youth/young people. Geography Compass, 2(5), pp. 1659-1680.

García, Javier (2013). Enfoque etnográfico y bachilleratos populares: negociaciones entre el tiempo escolar y la autogestión de los aprendizajes. X Jornadas de Sociología. Facultad de Ciencias Sociales, Universidad de Buenos Aires, Buenos Aires.

Glaser, Barney y Strauss, Anselm (1967). The discovery of grounded theory. Strategies of qualitative research. New York: Aldine.

Gentile, Ma. Florencia (2017). Biografias callejeras: cursos de vida de jóvenes en condiciones de desigualdad. Argentina: Grupo Editor Universitario.

Gough, Katherine (2008). 'Moving around': the social and spatial mobility of youth in Lusaka. Geografiska Annaler: Series B, Human Geography, 90(3), pp. 243-255.

Gough, Katherine y Franch Mónica (2005). Spaces of the street: socio-spatial mobility and exclusion of youth in Recife. Children's Geographies, 3(2), pp. 149-166.

Gutiérrez, Andrea y Reyes, Malena (2017). Mujeres entre la libertad y la obligación. Prácticas de movilidad cotidiana en el Gran Buenos Aires. Revista Transporte y Territorio, pp. 147-166.

Gutiérrez, Andrea (2012). ¿Qué es la movilidad? Elementos para (re) construir las definiciones básicas del campo del transporte. Bitácora, $\mathrm{N}^{\mathrm{o}} 21$, Vol. 2, pp. 61-74.

Gutiérrez, Andrea (2009). La movilidad de la metrópolis desigual: el viaje a la salud pública y gratuita en la periferia de Buenos Aires. XII Encuentro de Geógrafos de América Latina. Montevideo.

Infantino, Julieta (2011). Cultura, Jóvenes y Políticas en disputa. Prácticas circenses en la ciudad de Buenos Aires (Tesis de Doctorado). Facultad de Filosofía y Letras, Universidad de Buenos Aires, Buenos Aires. 
Jensen, Ole, Sheller, Mimi y Wind, Simon (2014). Together and Apart: Affective Ambiences and Negotiation in Families' Everyday Life and Mobility. Mobilities, pp. 1-20.

Jirón, Paola (2017). Planificación urbana y del transporte a partir de relaciones de interdependencia y movilidad del cuidado. En María N. Rico y Olga Segovia (eds.), ¿Quién cuida en la ciudad? Aportes para políticas urbanas de igualdad. Chile: CEPAL, pp. 403-430.

Jirón, Paola y Zunino Singh, Dhan (2017). Dossier. Movilidad Urbana y Género: experiencias latinoamericanas. Revista Transporte y Territorio. No 16, pp. 1-8.

Jirón, Paola, Lange, Carlos y Bertrand, María (2010). Exclusión y desigualdad espacial: Retrato desde la movilidad cotidiana. INVI, 25(68), pp. 15-57.

Kaufmann, Vincent; Bergman, Manfred y Joye, Dominique (2004). Motility: Mobility as Capital. International Journal of Urban and Regional Research, 28(4), pp. 745-756.

Kessler, Gabriel (2015). El sentimiento de inseguridad: sociología del temor al delito. Buenos Aires: Siglo Veintiuno Editores.

Maceira, Verónica (2012). Notas para una caracterización del Área Metropolitana de Buenos Aires. Buenos Aires: Instituto del Conurbano, Universidad Nacional de General Sarmiento. Recuperado de: http://www.observatoriodasmetropoles.net/download/01/notas_regi $\% \mathrm{C} 3$ \%B3n_metropolitana_de_Buenos\%20Aires.pdf

Machado, Mercedes; Mantiñán, Luciano y Grinberg, Silvia (2016). Relatos de infancias: nacer y vivir en las villas del sur global. Cartografía y devenir de la subjetividad en las sociedades contemporáneas. Última década, 24(45), 140-157.

Martuccelli, Danilo (2007). Cambio de rumbo. La sociedad a escala del individuo. Santiago de Chile: LOM.

Miranda, Ana (2008). Los jóvenes, la educación secundaria y el empleo a principios del siglo XXI. Revista de Trabajo, 4 (6), pp.185-198.

Pérez, Pablo; Deleo, Camila y Fernández Massi, Mariana (2013). Desigualdades sociales en trayectorias laborales de jóvenes en la Argentina, Revista Latinoamericana de Población, 7(13), pp. 61-89.

Saraví, Gonzalo (2014). Youth experience of urban inequality: space, class, and gender in Mexico. In: WYN, Johanna; CAHILL, Helen (Eds.). Handbook of childhood and youth. Singapore: Springer, pp. 1-11.

Saraví, Jorge (2012). Skate, espacios urbanos y jóvenes en la ciudad de La Plata (Tesis de Maestría en Educación Corporal). Facultad de Humanidades y Ciencias de la Educación, UNLP. 
232 "No me agrada viajar". Moverse en la ciudad como desafío cotidiano para jóvenes de barrios populares de Buenos Aires

Sheller, Mimi y Urry, John (2016). Mobilizing the new mobilities paradigm. Applied Mobilities, 1(1), pp. 10-25.

Scribano, Adrián (2012). Sociología de los cuerpos/emociones. Revista Latinoamericana de Estudios sobre Cuerpos, Emociones y SociedadRELACES, 10(4), pp. 91-111.

Scribano, Adrián (2011). Hacia unas ciencias sociales del Sur. Pensar las prácticas autonómicas entre el imperialismo, la dependencia y el colonialismo. Revista Pensamiento Plural, 8, pp. 11-36.

Scribano, Adrián (2010). Primero hay que saber sufrir!!! Hacia una sociología de la 'espera' como mecanismo de soportabilidad social. Sensibilidades en juego: miradas múltiples desde los estudios sociales del cuerpo y las emociones. Córdoba: CEA-CONICET, pp. 169-194.

Segura, Ramiro (2017). Desacoples entre desigualdades sociales, distribución del ingreso y patrones de urbanización en ciudades latinoamericanas. Reflexiones a partir de la Región Metropolitana de Buenos Aires. Revista CS, 21, pp. 15-39.

Skelton, Tracey (2013). Young people's urban im/mobilities: Relationality and identity formation. Urban Studies, 50(3), pp. 467-483.

Tapia, Silvia (2016). Salir, recorrer, permanecer. Movilidades cotidianas de jóvenes que realizan actividades artísticas y deportivas en barrios populares de la Ciudad de Buenos Aires. Argumentos. Revista de Crítica Social, (18), pp. 367-394.

Vasilachis, Irene (coord.). (2012). Estrategias de investigación cualitativa. Barcelona: Ed. Gedisa. 


\section{ANEXO I}

\section{DATOS DE LOS ENTREVISTADOS}

\begin{tabular}{|c|c|c|c|c|c|c|c|c|}
\hline Nombre & Institución & Nació en & Edad & $\begin{array}{c}\text { Reside } \\
\text { en }\end{array}$ & Escolaridad & Hijos & $\begin{array}{c}\text { Convive } \\
\text { con }\end{array}$ & $\begin{array}{c}\text { Condición } \\
\text { laboral }\end{array}$ \\
\hline Ramona & $\begin{array}{l}\text { Bachillerato } \\
\text { Popular }\end{array}$ & Argentina & 23 & GBA/sur & $\begin{array}{l}\text { Secundario } \\
\text { en curso }\end{array}$ & 3 hijos & $\begin{array}{l}\text { Pareja e } \\
\text { hijos }\end{array}$ & $\begin{array}{l}\text { Trabajo } \\
\text { doméstico. } \\
\text { Plan social }\end{array}$ \\
\hline Alberto & $\begin{array}{l}\text { Bachillerato } \\
\text { Popular }\end{array}$ & Argentina & 21 & GBA/sur & $\begin{array}{l}\text { Secundario } \\
\text { en curso }\end{array}$ & No tiene & \begin{tabular}{|l|} 
Padres, \\
hermanos \\
y \\
sobrinos \\
\end{tabular} & $\begin{array}{l}\text { Desemplea } \\
\text { do. Plan } \\
\text { social }\end{array}$ \\
\hline Carla & $\begin{array}{l}\text { Bachillerato } \\
\text { Popular }\end{array}$ & Argentina & 24 & GBA/sur & $\begin{array}{l}\text { Secundario } \\
\text { en curso }\end{array}$ & 1 hija & Hija & $\begin{array}{l}\text { Trabajo } \\
\text { informal }\end{array}$ \\
\hline Juan & $\begin{array}{l}\text { Bachillerato } \\
\text { Popular }\end{array}$ & Perú & 21 & $\begin{array}{l}\text { Villa- } \\
\text { CABA/ } \\
\text { sur }\end{array}$ & $\begin{array}{l}\text { Secundario } \\
\text { en curso }\end{array}$ & Un hijo & $\begin{array}{l}\text { Pareja y } \\
\text { hermano }\end{array}$ & $\begin{array}{l}\text { Trabaja en } \\
\text { un } \\
\text { programa } \\
\text { social }\end{array}$ \\
\hline Fatu & $\begin{array}{l}\text { Bachillerato } \\
\text { Popular }\end{array}$ & Paraguay & 18 & $\begin{array}{l}\text { Villa- } \\
\text { CABA/ } \\
\text { sur } \\
\end{array}$ & $\begin{array}{l}\text { Secundario } \\
\text { en curso }\end{array}$ & $\begin{array}{l}\text { Embarazo } \\
\text { en curso }\end{array}$ & \begin{tabular}{|l|} 
Pareja, \\
madre, \\
padrastro.
\end{tabular} & $\begin{array}{l}\text { Trabajo } \\
\text { doméstico }\end{array}$ \\
\hline Víctor & Circo & Argentina & 19 & $\mathrm{GBA} / \mathrm{sur}$ & $\begin{array}{l}\text { Secundario } \\
\text { completo }\end{array}$ & No tiene & \begin{tabular}{|l|} 
Padre, \\
madre y \\
hermanos
\end{tabular} & $\begin{array}{l}\text { Trabajos } \\
\text { informales }\end{array}$ \\
\hline Tincho & Circo & Argentina & 18 & CABA sur & $\begin{array}{l}\text { Secundario } \\
\text { en curso }\end{array}$ & No tiene & \begin{tabular}{|l|} 
Madre y \\
hermanos
\end{tabular} & $\begin{array}{l}\text { Pasantía } \\
\text { temporal }\end{array}$ \\
\hline Mariana & Circo & Argentina & 17 & $\begin{array}{l}\text { Villa- sur } \\
\text { de CABA }\end{array}$ & $\begin{array}{l}\text { Secundario } \\
\text { en curso }\end{array}$ & No tiene & $\begin{array}{l}\text { Padres y } \\
\text { hermanos }\end{array}$ & $\begin{array}{l}\text { Trabaja en } \\
\text { centro } \\
\text { barrial }\end{array}$ \\
\hline Jorge & COP & atina & 18 & $\begin{array}{l}\text { Villa- } \\
\text { CABA/ } \\
\text { sur }\end{array}$ & $\begin{array}{l}\text { Secundario } \\
\text { incompleto }\end{array}$ & No tiene & $\begin{array}{l}\text { Abuelos, } \\
\text { madre y } \\
\text { hermanos }\end{array}$ & No trabaja \\
\hline $\mathrm{Liz}$ & COP & Argentina & 21 & $\begin{array}{l}\text { Villa- } \\
\text { CABA/ } \\
\text { sur }\end{array}$ & $\begin{array}{l}\text { Secundaria } \\
\text { completa }\end{array}$ & Un hijo & $\begin{array}{l}\text { Madre e } \\
\text { hija }\end{array}$ & No trabaja \\
\hline Miguel & $\begin{array}{l}\text { Escuela } \\
\text { Secundaria }\end{array}$ & Argentina & 14 & $\begin{array}{l}\text { GBA- } \\
\text { oeste }\end{array}$ & $\begin{array}{l}\text { Secundario } \\
\text { en curso }\end{array}$ & No tiene & \begin{tabular}{|l} 
Madre, \\
padrastro \\
y \\
hermano \\
\end{tabular} & No trabaja \\
\hline Pecosa & \begin{tabular}{|l|} 
Escuela \\
Secundaria \\
\end{tabular} & Argentina & 16 & $\begin{array}{l}\text { GBA- } \\
\text { oeste }\end{array}$ & $\begin{array}{l}\text { Secundario } \\
\text { en curso }\end{array}$ & No tiene & Madre & No trabaja \\
\hline Fernanda & $\begin{array}{l}\text { Escuela } \\
\text { Secundaria }\end{array}$ & Argentina & 17 & $\begin{array}{l}\text { GBA- } \\
\text { oeste }\end{array}$ & $\begin{array}{l}\text { Secundario } \\
\text { en curso }\end{array}$ & No tiene & $\begin{array}{l}\text { Madre y } \\
\text { hermanos }\end{array}$ & No trabaja \\
\hline Susana & \begin{tabular}{|l|} 
Escuela \\
Secundaria
\end{tabular} & Argentina & 16 & $\begin{array}{l}\text { GBA- } \\
\text { oeste }\end{array}$ & $\begin{array}{l}\text { Secundario } \\
\text { en curso }\end{array}$ & No tiene & $\begin{array}{l}\text { Madre y } \\
\text { hermano }\end{array}$ & No trabaja \\
\hline
\end{tabular}

FECHA DE RECEPCIÓN: NOVIEMBRE 2017.

FECHA DE ACEPTACIÓN: MARZO 2018. 